\title{
Representation of the Medial-Like Algebras
}

\author{
Amir Ehsani \\ Department of Mathematics, Mahshahr Branch, Islamic azad University, Mahshahr, Iran. \\ a.ehsani@mahshahriau.ac.ir, amirehsany1978@gmail.com
}

\section{Abstract}

In this paper we characterize the regular medial algebras, the paramedial n-ary groupoids with a regular element, the paramedial algebras with a regular element and the regular paramedial algebras. Also, we characterize paramedial, co-medial and co-paramedial pairs of quasigroup operations and paramedial, co-medial and co-paramedial algebras with the quasigroup operations.

\section{Introduction}

An algebra, $A=(A, F)$, (without nullary operations) is called medial (entropic, abelian) if it satisfies the identity of mediality:

$$
\begin{gathered}
g\left(f\left(x_{11}, \ldots, x_{n 1}\right), \ldots, f\left(x_{1 m}, \ldots, x_{n m}\right)\right)= \\
f\left(g\left(x_{11}, \ldots, x_{1 m}\right), \ldots, g\left(x_{n 1}, \ldots, x_{n m}\right)\right),
\end{gathered}
$$

for every n-ary $f \in F$ and m-ary $g \in F[5]$. The n-ary operation, $f$, is called idempotent if $f(x, \ldots, x)=x$, for every $x \in A$. The algebra $A=(A, F)$ is called idempotent, if every operation $f \in F$ is idempotent. An idempotent medial algebra is a mode [10.

Let $g$ and $f$ be m-ary and n-ary operations on the set, $A$. We say that the pair of operations, $(f, g)$, is medial (entropic), if the identity (1) holds in the algebra, $A=(A, f, g)$.

We say that the pair of operations, $(f, g)$, is paramedial (or paraentropic), if the following identity holds in the algebra, $A=(A, f, g)$ :

$$
\begin{gathered}
g\left(f\left(x_{11}, \ldots, x_{n 1}\right), \ldots, f\left(x_{1 m}, \ldots, x_{n m}\right)\right)= \\
\quad f\left(g\left(x_{n m}, \ldots, x_{n 1}\right), \ldots, g\left(x_{1 m}, \ldots, x_{11}\right)\right) .
\end{gathered}
$$

An algebra, $A=(A, F)$, (without nullary operations) is called paramedial if every pair of operations, $f, g \in F$, (not necessarily distinct) is paramedial.

Paramedial groupoids and paramedial quasigroups were studied in [1, 9, 11].

Let $g$ and $f$ be n-ary operations on the set, $A$. We say that the pair of n-ary operations, $(f, g)$, is co-medial, if the following identity holds in the algebra, $A=(A, f, g)$ :

$$
\begin{gathered}
g\left(f\left(x_{11}, \ldots, x_{n 1}\right), \ldots, f\left(x_{1 n}, \ldots, x_{n n}\right)\right)= \\
g\left(f\left(x_{11}, \ldots, x_{1 n}\right), \ldots, f\left(x_{n 1}, \ldots, x_{n n}\right)\right) .
\end{gathered}
$$

The pair of n-ary operations, $(f, g)$, is co-paramedial, if the following identity holds in the algebra, $(A, f, g)$ :

$$
\begin{gathered}
g\left(f\left(x_{11}, \ldots, x_{n 1}\right), \ldots, f\left(x_{1 n}, \ldots, x_{n n}\right)\right)= \\
g\left(f\left(x_{n n}, \ldots, x_{n 1}\right), \ldots, f\left(x_{1 n}, \ldots, x_{11}\right)\right) .
\end{gathered}
$$

An algebra, $A=(A, F)$, is called a co-medial (co-paramedial) algebra, if every pair of the operations, $f, g \in F$, with the same arity is co-medial (co-paramedial).

In other words, the algebra, $A$, is medial (paramedial, co-medial, or co-paramedial) if it satisfies the hyperidentity of mediality (paramediality, co-mediality, or co-paramediality) [7, 8, 6 . 


\section{Main Results}

Let $A=(A, F)$ be an algebra and $f \in F$. We say that the element $e$, is the unit for the operation $f \in F$, if: $f(x, e, \ldots, e) \approx f(e, x, e, \ldots, e) \approx \ldots \approx f(e, \ldots, e, x) \approx x$, for every $x \in A$. The element $e$, is a unit for the algebra $(A, F)$, if it is a unit for every operation, $f \in F$. The element $e$, is idempotent for the operation $f$, if: $f(e, \ldots, e)=e$. We say that the element $e$, is idempotent for the algebra $(A, F)$, if it is an idempotent for every operation $f \in F$.

Definition 2.1. Let $(f, g)$ be a pair of m-ary and n-ary operations of the algebra, $(A, F)$. For any element $e$ of $A$, let $\alpha_{1}, \ldots, \alpha_{m}$ be mappings of $A$ into $A$ defined by

$$
\alpha_{i}: x \mapsto f(e, \ldots, e, x, e, \ldots, e),
$$

with $x$ at the i-th place. We call $\alpha_{i}$ the $i$-th translation by $e$ with respect to $f$. An element $e$ is called $i$-regular with respect to $f$ if $\alpha_{i}$ is a bijection. An element $e$, is called i-regular for the pair operation, $(f, g)$, if it is an i-regular with respect to the both operations $f$ and $g$. The element $e$, is called i-regular for the algebra $(A, F)$, if it is an i-regular element for every operation $f \in F$.

The element $e$ is called regular with respect to the $n$-ary operation $f \in F$, if $e$ is an i-regular element with respect to $f$ for every $1 \leq i \leq n$. The element $e$ is a regular element of the algebra $(A, F)$, if $e$ is a regular element with respect to the every operation $f \in F$.

Theorem 2.2. Let $(A, F)$ be a medial algebra with the idempotent element $e$ which is $i$ - and $j$-regular element of $(A, F)$ for fixed $i$ and $j(i \neq j)$, then there exists a commutative semigroup $(A,+)$ with the unit element $e$, such that every operation $f \in F$ has the following linear representation:

$$
f\left(x_{1}, \ldots, x_{m}\right)=\gamma_{1} x_{1}+\cdots+\gamma_{m} x_{m},
$$

where $\gamma_{1}, \ldots, \gamma_{m}$, are pairwise commuting endomorphisms of $(A,+), m \geq 2$. Furthermore, $\gamma_{i}, \gamma_{j}$ are automorphisms.

Definition 2.3. Let $f$ be an $m$-ary operation and $J$ be a non-empty subset of $\{1,2, \ldots, m\}$, we will say that the element $e$ is $J$-regular with respect to the operation $f$, if $e$ is a $j$-regular element with respect to $f$, for all $j \in J$. The element $e$ is $J$-regular element for the algebra $(A, F)$, if $e$ is a $j$-regular element with respect to every $f \in F$, for all $j \in J$, where $m=$ $\min \{|g| \mid g \in F\}$, and $m \geq 2$.

Definition 2.4. Let $f, g \in F$ be m-ary and n-ary operations $(m \leq n), J \subseteq\{1,2, \ldots, m\}$ (where $J$ contains at leas two elements) and $a_{1}, \ldots, a_{i-1}, a_{i+1}, \ldots, a_{m}, \ldots, a_{n}$ are $J$-regular elements of the algebra $(A, f, g)$. The pair operation $(f, g)$ is $(i, J)$-regular pair operation (where $i \in J$ ), if for every $x \in A$ we have the following equation:

$$
f\left(a_{1}, \ldots, a_{i-1}, x, a_{i+1}, \ldots, a_{m}\right)=g\left(a_{1}, \ldots, a_{i-1}, x, a_{i+1}, \ldots, a_{n}\right)
$$

The pair operation $(f, g)$ is a $J$-regular pair operation if $(f, g)$ is $(i, J)$-regular for every $i \in J$. The pair operation $(f, g)$ is a regular pair operation if $(f, g)$ is $J$-regular pair operation for some $J \subseteq\{1,2, \ldots, m\}$ (where $J$ contains at leas two elements).

An algebra $(A, F)$ is called a regular algebra if every pair operation of $(A, F)$ be a regular pair operation.

Theorem 2.5. Let $(A, f, g)$ be a regular medial algebra with $m$-ary operation $f$ and $n$-ary operation $g(m \leq n)$, then there is a commutative semigroup with an unit element $(A,+)$, such that

$$
\begin{array}{r}
f\left(x_{1}, \ldots, x_{m}\right)=\gamma_{1} x_{1}+\cdots+\gamma_{m} x_{m}+d_{1}, \\
g\left(x_{1}, \ldots, x_{n}\right)=\lambda_{1} x_{1}+\cdots+\lambda_{n} x_{n}+d_{2},
\end{array}
$$


where, $d_{1}, d_{2}$ are fixed regular elements in $(A,+)$ and $\gamma_{1}, \ldots, \gamma_{m}, \lambda_{1}, \ldots, \lambda_{n}$, are commuting automorphisms of the semigroup $(A,+)$.

Corollary 2.6. Let $(A, F)$ be a regular medial algebra, then there exists a commutative semigroup $(A,+)$, such that every operation $f \in F$ has the following representation:

$$
f\left(x_{1}, \ldots, x_{m}\right)=\gamma_{1} x_{1}+\cdots+\gamma_{m} x_{m}+d,
$$

where $d$ is a fixed regular element in $(A,+)$ and $\gamma_{1}, \ldots, \gamma_{m}$ are commuting automorphisms of the semigroup $(A,+)$.

Corollary 2.7. 4 . If $(Q, f)$ is a medial n-ary quasigroup, then there exists an abelian group, $(Q,+)$, such that

$$
f\left(x_{1}, \ldots, x_{m}\right)=\alpha_{1} x_{1}+\cdots+\alpha_{m} x_{m}+d,
$$

where $\alpha_{i} \in \operatorname{Aut}(Q,+)$, are pairwise commute, and $d \in Q$.

There exist various algebraic characterizations of different classes of n-ary operations (see, for instance, [2]).

Theorem 2.8. Let $(A, f)$ be a paramedial n-ary groupoid such that $A$ contains an n-ary regular subgroupoid, then there is a commutative semigroup with unit element $(A,+)$, such that

$$
f\left(x_{1}, \ldots, x_{n}\right)=\gamma_{1} x_{1}+\cdots+\gamma_{n} x_{n}+d,
$$

where, $d$ is a fixed regular element in $(A,+)$ and $\gamma_{1}, \ldots, \gamma_{n}$, are automorphisms of the semigroup $(A,+), n \geq 2$. Moreover: $\gamma_{i} \gamma_{j}=\gamma_{n-j+1} \gamma_{n-i+1}$, for $n>2, i, j=1, \ldots, n$, and for $n=2$ we have: $\gamma_{1}^{2}=\gamma_{2}^{2}$.

Corollary 2.9. Let $(Q, f)$ be a paramedial n-ary quasigroup, then there exists an abelian group $(Q,+)$, such that

$$
f\left(x_{1}, \ldots, x_{n}\right)=\gamma_{1} x_{1}+\cdots+\gamma_{n} x_{n}+d,
$$

where, $d$ is a fixed element in $(Q,+)$ and $\gamma_{1}, \ldots, \gamma_{n}$, are automorphisms of the abelian group $(Q,+), n \geq 2$. Moreover: $\gamma_{i} \gamma_{j}=\gamma_{n-j+1} \gamma_{n-i+1}$, for $n>2, i, j=1, \ldots, n$, and for $n=2$ we have: $\gamma_{1}^{2}=\gamma_{2}^{2}$.

Theorem 2.10. Let $(A, F)$ be a paramedial algebra with the regular idempotent element $e$, then there exists a commutative semigroup $(A,+)$ with the unit element $e$, such that every operation $f \in F$ has the following linear representation

$$
f\left(x_{1}, \ldots, x_{m}\right)=\alpha_{1} x_{1}+\cdots+\alpha_{m} x_{m},
$$

where $\alpha_{1}, \ldots, \alpha_{m}$, are pairwise commuting automorphisms of $(A,+), m \geq 2$.

Theorem 2.11. Let $(A, f, g)$ be a regular paramedial algebra with $m$-ary operation $f$ and $n$-ary operation $g$, then there is a commutative semigroup with unit element $(A,+)$, such that

$$
\begin{array}{r}
f\left(x_{1}, \ldots, x_{m}\right)=\gamma_{1} x_{1}+\cdots+\gamma_{m} x_{m}+d_{1}, \\
g\left(x_{1}, \ldots, x_{n}\right)=\lambda_{1} x_{1}+\cdots+\lambda_{n} x_{n}+d_{2},
\end{array}
$$

where, $d_{1}, d_{2}$ are fixed regular elements in $(A,+)$ and $\gamma_{1}, \ldots, \gamma_{m}, \lambda_{1}, \ldots, \lambda_{n}$, are commuting automorphisms of the semigroup $(A,+)$.

Theorem 2.12. Let $(Q, F)$ is a binary paramedial algebra with quasigroup operations, then there exists an abelian group $(Q,+)$, such that every operation, $f_{i} \in F$, is represented by the following rule:

$$
f_{i}(x, y)=\varphi_{i}(x)+\psi_{i}(y)+c_{i},
$$


where $c_{i} \in Q$ and $\varphi_{i}, \psi_{i} \in \operatorname{Aut}(Q,+)$, such that: $\varphi_{i} \varphi_{j}=\psi_{j} \psi_{i}, \varphi_{i} \psi_{j}=\varphi_{j} \psi_{i}, \psi_{i} \varphi_{j}=\psi_{j} \varphi_{i}$. The group, $(Q,+)$, is unique up to isomorphisms.

Theorem 2.13. Let $(Q, F)$ is a binary co-medial algebra with quasigroup operations, then there exists an abelian group $(Q,+)$, such that every operation, $f_{i} \in F$, is represented by the following rule:

$$
f_{i}(x, y)=\varphi_{i}(x)+\psi_{i}(y)+c_{i},
$$

where $c_{i} \in Q$ and $\varphi_{i}, \psi_{i} \in \operatorname{Aut}(Q,+)$, such that $\varphi_{i} \psi_{j}=\psi_{i} \varphi_{j}$. The group, $(Q,+)$, is unique up to isomorphisms.

Theorem 2.14. Let $(Q, F)$ is a binary co-paramedial algebra with quasigroup operations, then there exists an abelian group $(Q,+)$, such that every operation, $f_{i} \in F$, is represented by the following rule:

$$
f_{i}(x, y)=\varphi_{i}(x)+\psi_{i}(y)+c_{i},
$$

where $c_{i} \in Q$ and $\varphi_{i}, \psi_{i} \in \operatorname{Aut}(Q,+)$, such that $\varphi_{i} \varphi_{j}=\psi_{i} \psi_{j}$. The group, $(Q,+)$, is unique up to isomorphisms.

Further description of the contents of this section are available in $[3]$.

\section{References}

[1] J. Cho, J. ježek, and T. Kepka. Paramedial groupoids. Czechoslavak Math. J., 49(2):227-240, 1999.

[2] W. A. Dudek and V. S. Trokhimenko. Algebras of multiplace functions. Kremenchug Press, 2010.

[3] A. Ehsani and Yu. M. Movsisyan. Linear representation of medial-like algebras. Communications in Algebra, 41(9), 2013. DOI:10.1080/00927872.2012.688154. Be appear.

[4] T. Evans. Abstract mean value. Duke Math J., 30:331-349, 1963.

[5] A. G. Kurosh. General Algebra. M. Nauka, 1974. Russian.

[6] Yu. M. Movsisyan. Introduction to the theory of algebras with hyperidentities. Yerevan State University Press, Yerevan, Armenia, 1986. Russian.

[7] Yu. M. Movsisyan. Hyperidentities and hypervarieties in algebras. Yerevan State University Press, Yerevan, Armenia, 1990. Russian.

[8] Yu. M. Movsisyan. Hyperidentities in algebras and varieties. Uspekhi Math. Nauk, 53:61-114, 1998. English translation in: Russian Math. Surveys 53 (1998), No1, pp. 57-108.

[9] P. Němec and T. Kepka. T-quasigroups i. Acta Univ. Carolin. Math. Phys., 12(1):39-49, 1971.

[10] A. Romanowska and J. D. H. Smith. Modes. World Scientific Press, 2002.

[11] V. A. Shcherbakov and D. I. Pushkashu. On the structure of finite paramedial quasigroups. Comment. Math. Univ. Carolin., 51(2):357-370, 2010. 